\title{
Evaluación de un método manual para producir plasma rico en plaquetas-puro (P-PRP) en conejos: estudio hematológico
}

\author{
Evaluation of a manual method for producing pure-platelet rich plasma (P-PRP) in rabbits: \\ hematological study
}

JC González, C López, JU Carmona*

Grupo de Investigación Terapia Regenerativa, Departamento de Salud Animal, Universidad de Caldas, Manizales, Caldas, Colombia.

\section{SUMMARY}

\begin{abstract}
The objective of this study was to evaluate a manual method to produce two kinds of pure-platelet rich plasma (P-PRP-A and P-PRP-B) in rabbits. Both P-PRP were obtained by drawing blood from the jugular vein into tubes containing sodium citrate as anticoagulant and, centrifuged at $259 \mathrm{~g}$ for $6 \mathrm{minutes}$. The number of concentrated platelets in the P-PRP-A was $716.14 \mathrm{X} 10^{3} / \mu \mathrm{L}$ and $630 \mathrm{X} 10^{3} / \mu \mathrm{L}$ for P-PRP-B. Cell counts were significantly different $(\mathrm{P}<0.05)$ between whole blood and PRP fractions A and B, for RBC counts $\left(10^{6} / \mu \mathrm{L}\right)$, WBC $\left(10^{3} / \mu \mathrm{L}\right)$, PLT $\left(10^{3} / \mu \mathrm{L}\right)$ and PDW $(\%)$ but not for MPV (fL). Gender differences were found in P-PRP-A for RBC $\left(10^{6} / \mu \mathrm{L}\right)$, which was higher in males, and the MPV (fL), which was higher in females. In fraction P-PRP-B a difference was found for MPV (fL), which was higher in females. The PDW (\%) was higher in males compared with females. The platelet collection efficiency was $20.76 \%$ for P-PRP-A and $18.27 \%$ for P-PRP-B. The platelet concentration considering whole blood was $186.92 \%$ for P-PRP-A and $164.44 \%$ for P-PRP-B. The protocol described in this study represents a simple method to obtain P-PRP in rabbits for experimental purposes and even for therapeutic use.
\end{abstract}

Key words: rabbit, platelet concentrate, tube method, cellular study, regenerative therapy.

\section{RESUMEN}

El objetivo de este estudio fue evaluar un método manual para producir dos clases de plasma rico en plaquetas-puro (P-PRP-A y P-PRP-B) en conejos. Ambos P-P-PRP se obtuvieron por extracción de sangre de la vena yugular en tubos con citrato de sodio como anticoagulante y centrifugados a $259 \mathrm{~g}$ durante 6 minutos. El número de plaquetas concentradas en la fracción P-PRP-A fue de 716,14 X $10^{3} / \mu \mathrm{L}$ ) y 630 X $10^{3} / \mu \mathrm{L}$ para la fracción P-PRP-B. Para los conteos celulares entre sangre entera y las fracciones P-PRP-A y P-PRP-B, se encontraron diferencias estadísticamente significativas $(\mathrm{P}<0,05)$ entre los recuentos de RBC $\left(10^{6} / \mu \mathrm{L}\right)$, WBC $\left(10^{3} / \mu \mathrm{L}\right)$, PLT $\left(10^{3} / \mu \mathrm{L}\right)$ y PDW $(\%)$ pero no para MPV (fL). Se encontraron diferencias entre sexos para RBC $\left(10^{6} / \mu \mathrm{L}\right)$, que fue mayor en machos, y el MPV (fL) (mayor en hembras) para la fracción P-PRP-A. En La fracción P-PRP-B se encontró diferencia para MPV (fL) (mayor en hembras). El PDW (\%) fue mayor en los machos respecto al hemograma. La eficiencia de colección de plaquetas para la fracción P-PRP-A fue de 20,76\% y para la fracción P-PRP-B de 18,27\%. La concentración de plaquetas respecto a la sangre entera fue de 186,92\% para la fracción P-PRP-A y de 164,44\% para la fracción P-PRP-B. El protocolo descrito en este estudio representa un método sencillo para obtener P-PRP en conejos con finalidades experimentales e incluso, terapéuticas.

Palabras clave: conejo, concentrado de plaquetas, método del tubo, estudio celular, terapia regenerativa.

\section{INTRODUCCIÓN}

Tanto seres humanos como caballos se pueden ver afectados por enfermedades del aparato locomotor, tales como tendinopatías. Estas afecciones son de difícil tratamiento (Andres y Murrell 2008) y la medicación clásica como los analgésicos no esteroidales (AINES) o los corticoesteroides solo alivian el dolor (efecto sintomático), no detienen el proceso degenerativo y pueden ser catabólicos sobre sobre la matriz extra celular (ECM) de los tendones afectados (Rees y col 2006).

Aceptado: 16.05 .2013 .

* Calle 65 No 26-10, Manizales, Caldas, Colombia; carmona@ucaldas.edu.co
El uso de terapias autólogas basadas en células y factores de crecimiento (GF) como el plasma rico en plaquetas (PRP), se ha convertido en una opción terapéutica para el tratamiento de tendinopatías en seres humanos y caballos (Anitua y col 2004, Arguelles y col 2006, Carmona y col 2009). El PRP es una fuente importante de GF con propiedades anti-inflamatorias, anabólicas y angiogénicas. Los principales GF contenidos en esta sustancia son el GF transformante beta 1 (TGB- $\beta_{1}$ ), el GF parecido a la insulina tipo 1 (IGF-I) y el GF derivado de las plaquetas (PDGF), entre otros. Los GF están implicados de forma natural en los procesos de reparación de tejidos, tales como tendones y ligamentos (Molloy y col 2003). Al parecer la adición de GF y células mediante la inyección de PRP mejoran la reparación e incluso producen regeneración de los tendones afectados por lesiones traumáticas y degenerativas (Lyras y col 2010, Soomekh 2011). 
Existen 2 tipos generales de concentrados de plaquetas (PC) que pueden ser aplicados como tratamiento médico de afecciones tendinosas. Estos PC son obtenidos con anticoagulantes (como el citrato de sodio) y normalmente son activados (con gluconato de calcio, trombina u otra sustancia), antes de su inyección en el foco de la lesión. De manera general, y en función del número de leucocitos y plaquetas concentradas por $\mu \mathrm{L}$ de plasma, los PC se clasifican en PRP-puro (P-PRP) y plasma rico en plaquetas y leucocitos (L-PRP). El P-PRP puede presentar conteos plaquetarios hasta cuatro veces el recuento basal obtenido en sangre entera y conteos leucocitarios hasta 2 veces o menos respecto al conteo de estas células en sangre entera. El L-PRP puede presentar conteos plaquetarios 5 veces más altos respecto conteo de los mismos fragmentos citoplasmicos en sangre entera y conteos leucocitarios 3 veces o más altos respecto al recuento de estas células en sangre entera (Ehrenfest y col 2009).

En seres humanos (Soomekh 2011) y en caballos (Schnabel y col 2007, Carmona y col 2009) se ha observado que P-PRP y L-PRP tienen efectos clínicos benéficos en pacientes con tendinopatías. Sin embargo, hasta el momento no se sabe qué clase de PC (P-PRP o L-PRP) podría ser más benéfico como tratamiento de esta clase de afecciones locomotoras. Para resolver esta pregunta se hace necesario desarrollar una serie de investigaciones que incluyan estudios hematológicos, evaluación en modelos animales y en pacientes con enfermedad natural.

El conejo es uno de los modelos animales más utilizados para estudiar la fisiopatología de las tendinopatías y para evaluar los diversos efectos de fármacos convencionales o terapias regenerativas (Carpenter y Hankenson 2004, Lyras y col 2009, Lyras y col 2010). Debido al creciente interés sobre el uso del PRP como tratamiento regenerativo de afecciones tendinosas en seres humanos y caballos y a las ventajas que ofrece el conejo como modelo animal de tendinopatía, se hace necesario desarrollar protocolos para la obtención de P-PRP y L-PRP y su posterior evaluación en tendinopatía experimental. Según la literatura revisada, aunque se han descrito algunos métodos para producir PRP en conejos, no existen protocolos suficientemente estandarizados para concentrar plaquetas en esta especie. Cabe aclarar que esto implica una descripción adecuada del protocolo en términos de tiempo, fuerzas relativas de centrifugación (RCF o g), tipo de anticoagulante y características celulares del PC obtenido, entre otras.

El objetivo de esta investigación fue describir un protocolo manual para producir dos clases de P-PRP a partir de sangre leporina, evaluar la población celular concentrada y conocer el grado de activación plaquetaria en ambos PC y correlacionar los conteos celulares de ambos PC con los conteos de estas mismas células en sangre entera. La hipótesis del estudio fue que las plaquetas de conejos podrían ser concentradas mediante recolección de sangre entera en tubos con citrato de sodio $(3,2 \%)$ mediante una sola centrifugación.

\section{MATERIAL Y MÉTODOS}

\section{ANIMALES}

Se utilizaron 12 conejos Nueva Zelanda esqueléticamente maduros. Seis machos y seis hembras entre los 6 meses y un año de edad, con pesos comprendidos entre 3 a 4,2 Kg. Los animales fueron clínicamente sanos al momento de la toma de las muestras. Los conejos se alojaron en jaulas individuales con comida y agua a voluntad. Este estudio fue aprobado por el comité de ética para experimentación con animales de la institución de los autores.

\section{PROTOCOLO PARA OBTENER LAS DOS CLASES DE P-PRP}

Luego de anestesiar cada conejo con xilacina al $2 \%$ ( $5 \mathrm{mg} / \mathrm{Kg}$ Laboratorios Synthesis, Colombia) y ketamina al 10\% (30mg/Kg Laboratorio Merial, Francia). El tejido subcutáneo adyacente a una vena yugular fue infiltrado con $2 \mathrm{~mL}$ de lidocaína al $2 \%$ y posteriormente se realizó exteriorización de la vena mediante disección. La sangre fue extraída mediante punción de la vena yugular con un catéter mariposa $21 \mathrm{G}$ (Terumo, Leuven, Belgica). La cabeza de los animales fue mantenida colgada para facilitar la ingurgitación de la vena yugular. La herida fue suturada con 3 puntos simples de sutura no absorbible calibre 2-0.

La sangre de cada animal se depositó en 3 tubos de $4,5 \mathrm{ml}$ con $0,5 \mathrm{ml}$ de citrato de sodio (BD Vacutainer, Franklin Lakes NJ, USA). A una de las muestras se le realizó hemograma. El resto de tubos fue centrifugado a $259 \mathrm{~g}$ durante 6 minutos. La fracción de plasma obtenida fue arbitrariamente dividida en P-PRP-A y P-PRP-B. La fracción P-PRP-A fue considerada como el 50\% de la fracción plasmática inmediatamente superior a la interface leuco-plaquetaria ("buffy coat") y el 50\% del plasma superior al P-PRP-A fue considerado como P-PRP-B.

Las fracciones obtenidas (P-PRP-A y P-PRP-B) fueron analizadas por medio de hemograma automatizado por impedancia volumétrica (MEK 640, Nihon Kohden, Japón). Cada una de las muestras se analizó por duplicado. Los parámetros hematológicos evaluados fueron conteo de leucocitos (WBC $10^{3} / \mu \mathrm{L}$ ), eritrocitos (RBC $10^{6} / \mu \mathrm{L}$ ), plaquetas (PLT $10^{3} / \mu \mathrm{L}$ ), volumen plaquetario medio (MPV fL) y volumen de distribución plaquetario (PDW \%).

\section{ANÁLISIS ESTADÍSTICO}

Los parámetros hematológicos estudiados fueron analizados con un test de Kolmogorov-Smirnov $(\mathrm{P}<0,05)$ como prueba de normalidad. Una prueba de Levene $(\mathrm{P}>0,05)$ fue utilizada para el análisis de homogeneidad de varianzas. Las variables que presentaron distribución paramétrica se analizaron mediante una ANOVA de una vía y sus resultados se presentaron como media y desviación estándar. El análisis de las variables no paramétricas se realizó median- 
te una prueba de Kruskal-Wallis, los resultados se presentaron como mediana y rango intercuartílico. Como pruebas post-hoc se emplearon la prueba Games-Howell y la U de Mann-Whitney. Las comparaciones entre sexos fueron realizadas mediante una prueba $t$ para muestras independientes. Se realizó una correlación de Spearman para evaluar una posible asociación entre variables. Un valor de $\mathrm{P}<0,05$ fue aceptado como significativo para todas las pruebas.

La eficiencia de colección de plaquetas se determinó mediante la fórmula de Weibrich y col (Weibrich y col 2003):(volumen de APC x recuento de plaquetas en el APC/volumen de sangre entera $\mathrm{x}$ recuento de plaquetas en sangre entera) $\mathrm{x} 100$.

\section{RESULTADOS}

Los recuentos celulares de la sangre entera y de los P-PRP-A y P-PRP-B presentaron diferencia estadísticamente significativa $(\mathrm{P}<0,05)$ para las variables $\mathrm{RBC}\left(10^{6} \%\right.$ $\mu \mathrm{L}), \mathrm{WBC}\left(10^{3} / \mu \mathrm{L}\right), \operatorname{PLT}\left(10^{3} / \mu \mathrm{L}\right)$ y $\operatorname{PDW}(\%)$. La variable RBC $\left(10^{6} / \mu \mathrm{L}\right)$ mostró diferencia entre las fracciones P-PRP-A y P-PRP-B (cuadro 1). Los machos presentaron valores estadísticamente significativos más altos para el
MPV(fL) y el PDW(\%) en ambas fracciones, mientras que las hembras presentaron incremento del MPV(fL) en la fracción P-PRP-A (cuadro 2). Se encontró correlación negativa $\left(R_{S}=-0,731\right)$ entre el volumen de distribución plaquetaria y el recuento plaquetario (cuadro 3 ).

La eficiencia de colección de plaquetas para la fracción P-PRP-A fue de $20,76 \%$ y para la fracción P-PRP-B de $18,27 \%$. La concentración de plaquetas fue del 187\% para la fracción P-PRP-A y del 164\% para la fracción PPRP-B, respecto a la sangre entera.

\section{DISCUSIÓN}

Existen varios trabajos experimentales descritos en conejos en los que se han evaluado los PC como tratamiento de lesiones del aparato locomotor, en evaluación de implantes odontológicos y como pegante biológico (Findikcioglu y col 2009, Dong y col 2012, Lee y col 2012). En dichos trabajos se han empleado diversos métodos para concentrar plaquetas en esta especie. Sin embargo, existe una gran disimilitud entre los diferentes tipos de protocolos empleados, el anticoagulante usado y la clase de PRP obtenido. También se han mencionado diferentes formas

Cuadro 1. Resultados generales por grupo hematológico, P-PRP-A y P-PRP-B en Citrato.

General results by haematological group, P-PRP-A and P-PRP-B in Citrate.

\begin{tabular}{|c|c|c|c|}
\hline Variable & $\begin{array}{c}\text { Hemograma } \\
(n: 12)\end{array}$ & $\begin{array}{l}\text { P-PRP-A } \\
(n: 12)\end{array}$ & $\begin{array}{l}\text { P-PRP-B } \\
(n: 12)\end{array}$ \\
\hline $\mathrm{RBC} X 10^{6} / \mu \mathrm{L}$ & $6,49(0,51)^{\mathrm{a}}$ & $0,29(0,16)^{\mathrm{b}}$ & $0,09(0,02)$ \\
\hline $\mathrm{WBC} \times 10^{3} / \mu \mathrm{L}$ & $7,06(1,59)^{\mathrm{c}, \mathrm{d}}$ & $6,94(3,74)$ & $3,78(0,53)$ \\
\hline $\operatorname{PLT} \times 10^{3} / \mu \mathrm{L}$ & $383,13(88,19)^{\mathrm{c}, \mathrm{d}}$ & $716,14(219,39)$ & $630,0(157,16)$ \\
\hline MPV fL & $2,40(0,61)$ & $2,82(0,61)$ & $2,98(0,65)$ \\
\hline PDW \% & $19,74(1,42)^{\mathrm{a}}$ & $16,84(0,80)$ & $16,98(0,14)$ \\
\hline
\end{tabular}

Datos presentados como media y SD. a: P $<0,001$ con P-PRP-A, P-PRP-B; b: P < 0,05 con P-PRP-B; c: P $<0,05$ con P-PRP-A; d: P < 0,001 con P-PRP-B.

Cuadro 2. Resultados generales por sexo y grupo hematológico, P-PRP-A y P-PRP-B. General results for sex and haematological group, P-PRP-A and P-PRP-B.

\begin{tabular}{lcccccc}
\hline & \multicolumn{2}{c}{ Hemograma } & \multicolumn{2}{c}{ P-PRP-A } & \multicolumn{2}{c}{ P-PRP-B } \\
\cline { 2 - 6 } Variable & $\begin{array}{c}\text { Macho } \\
(n: 6)\end{array}$ & $\begin{array}{c}\text { Hembra } \\
(n: 6)\end{array}$ & $\begin{array}{c}\text { Macho } \\
(n: 6)\end{array}$ & $\begin{array}{c}\text { Hembra } \\
(n: 6)\end{array}$ & $\begin{array}{c}\text { Macho } \\
(n: 6)\end{array}$ & $\begin{array}{c}\text { Hembra } \\
(n: 6)\end{array}$ \\
\hline RBC X 10 $\mu \mathrm{L}^{*}$ & $6,60(1,81)$ & $6,38(1,39)$ & $0,03(0,16)^{\mathrm{a}}$ & $0,28(0,54)$ & $0,04(0,06)$ & $0,04(0,11)$ \\
WBC X10 ${ }^{3} \mu \mathrm{L}$ & $7,29(2,00)$ & $6,80(1,03)$ & $2,01(1,97)^{\mathrm{a}}$ & $6,73(4,55)$ & $2,50(1,45)$ & $2,29(1,20)$ \\
PLT X10 ${ }^{3} \mu \mathrm{L}^{*}$ & $397,00(363)$ & $405,00(246)$ & $493,50(482)$ & $655,50(750)$ & $555,50(323)$ & $597,00(567)$ \\
MPV(fL) & $2,31(0,67)$ & $2,50(0,56)$ & $2,41(0,59)^{\mathrm{a}}$ & $2,72(0,56)$ & $2,80(0,48)$ & $2,63(0,69)$ \\
PDW(\%) & $20,59(1,29)^{\mathrm{b}}$ & $18,81(0,91)$ & $18,01(1,03)^{\mathrm{a}}$ & $16,71(0,70)$ & $17,55(0,85)^{\mathrm{a}}$ & $16,58(0,61)$ \\
\hline
\end{tabular}

* Datos presentados como mediana (RI). P-PRP-A, Plasma rico en plaquetas puro fracción A y B. Letras minúsculas denotan diferencias significativas entre sexos dentro de cada grupo hematológico, mediante pruebas $\mathrm{T}$ y $\mathrm{K}-\mathrm{W}$ en variables paramétricas y no paramétricas respectivamente. a: $\mathrm{P}<0,05$ con hembra. b: $\mathrm{P}<0,01$ con hembra. 
Cuadro 3. Correlaciones generales de Spearman para las variables hematológicas WBC X $10^{3} \mu \mathrm{L}, \mathrm{RBC} \times 10^{6} \mu \mathrm{L}$, PLT $\mathrm{X} 10^{3} \mu \mathrm{L}, \mathrm{MPV}(\mathrm{fL})$ y $\mathrm{PDW}(\%)$.

General Spearman correlations for the haematological variables WBC X $10^{3} \mu \mathrm{L}, \mathrm{RBC} \times 10^{6} \mu \mathrm{L}$, PLT X10 $0^{3} \mu \mathrm{L}, \mathrm{MPV}(\mathrm{fL})$ y $\operatorname{PDW}(\%)$.

\begin{tabular}{lrrrr}
\hline & WBC & RBC & PLT & MPV \\
\hline WBC X $10^{3} \mu \mathrm{L}$ & - & - & - & - \\
RBC X $10^{6} \mu \mathrm{L}$ & $0,871 * *$ & - & - & - \\
PLT X $10^{3} \mu \mathrm{L}$ & & n.s. & - & - \\
MPV $(\mathrm{fL})$ & n.s. & n.s. & $0,336 * *$ & - \\
PDW $(\%)$ & $0,271 * *$ & $0,462 * *$ & $-0,731 * *$ & $-0,311 * *$ \\
\hline
\end{tabular}

** Correlación significativa al nivel 0,01 (dos colas); n.s.: no significativo.

de obtener la sangre de los conejos como la extracción intracardiaca, de la vena femoral y de las venas marginales y centrales de la oreja (Masago y col 2007, Findikcioglu y col 2009, Lee y col 2012). En el caso de esta investigación y después de realizar varias pruebas piloto, se pudo determinar que la mejor forma de extraer las cantidades de sangre requeridas para la preparación de los P-PRP fue mediante disección yugular, ya que con los otros métodos mencionados no se pudieron obtener volúmenes adecuados por pérdida de la presión al extraer la sangre y por la formación de coágulos. Para el conocimiento de los autores este es el primer reporte en el que se describe este método para obtener sangre en conejos.

Algunos investigadores se han basado en protocolos manuales desarrollados para concentrar plaquetas en humanos (Ishida y col 2007, Nagata y col 2010, Ohba y col 2012), los cuales usan la doble centrifugación con el incremento del número y tiempo de las revoluciones de centrifugación (cuadro 4). Estos métodos potencialmente podrían inducir activación temprana de las plaquetas con la subsiguiente liberación de los factores de crecimiento (Nagata y col 2010). Por otra parte, Lyras y col (2010), al igual que en nuestro estudio, reportaron un método de centrifugación simple para producir PRP en conejos. Sin embargo, ellos no describieron detalladamente su protocolo, puesto que no mencionaron el anticoagulante utilizado, el número de plaquetas y leucocitos concentrados, ni ningún parámetro hematológico para evaluar la integridad de las mismas, tales como el MPV (fL) y el PDW (\%).

Se decidió utilizar citrato de sodio en nuestro estudio, ya que es uno de los anticoagulantes más empleados para producir plasma rico en plaquetas (PRP). Se ha reportado el uso de varios anticoagulantes que revierten la activación plaquetaria y previenen la liberación temprana de los factores de crecimiento para producir PRP, como el ACD-A. Sin embargo, investigaciones no publicadas por los autores han demostrado que el citrato de sodio no compromete la concentración de factores de crecimiento en comparación con ACD-A. Otros anticoagulantes como la heparina y el EDTA pueden alterar la estructura de la plaqueta y causar activación temprana de la misma con lo que la calidad de los PRPs se ve alterada (White y Escolar 2000, Lei y col 2009).

Los PC no solo contienen plaquetas de manera estricta, sino que son una suspensión plasmática con glóbulos rojos y células blancas que potencialmente podrían influir en la calidad biológica de estas sustancias (Everts y col 2006). En el caso de este estudio, la fracción P-PRPB concentró una cantidad de $630 \times 10^{3} \mathrm{PLT} / \mu \mathrm{L}$ con una menor cantidad de leucocitos y eritrocitos que las obtenidas en la fracción P-PRP-A. A pesar de que los PC de este estudio presentaron una menor cantidad de plaquetas en comparación con un método de doble centrifugación (Nagata y col 2010) en el que se obtuvieron 1986 X $10^{3}$ $\mathrm{PLT} / \mu \mathrm{L}$, se puede considerar que el número de plaquetas del presente estudio podría ser suficiente para producir algún tipo de efecto, ya que en seres humanos y caballos con enfermedad natural se ha observado que la respuesta

Cuadro 4. Comparación de métodos utilizados para concentrar plaquetas en conejos. Comparison of methods used to concentrate platelets in rabbits.

\begin{tabular}{|c|c|c|c|c|c|c|c|}
\hline $\begin{array}{l}\text { Tipo de recuento } \\
\text { plaquetas }\end{array}$ & $\begin{array}{l}\text { Anticoa- } \\
\text { gulante }\end{array}$ & Plaquetas basal & $\begin{array}{l}\text { Protocolo de } \\
\text { centrifugado }\end{array}$ & r.p.m/tiempo & Plaquetas PRP & Sitio extracción & Autores \\
\hline Automático & $\mathrm{ACD}$ & $21,33 \times 10^{4} \mu \mathrm{L}$ & $\begin{array}{l}1^{\mathrm{a}} \text { vez } \\
2^{\mathrm{a}} \text { vez }\end{array}$ & $\begin{array}{c}800 / 15 \mathrm{~min} \\
2.000 / 10 \mathrm{~min}\end{array}$ & $104,45 \times 10^{4} \mu \mathrm{L}$ & Sin reporte & Ishida et al 2007 \\
\hline Manual & Cit $\mathrm{Na} 10 \%$ & $458 \times 10^{6} \mu \mathrm{L}$ & $\begin{array}{c}\text { Grupo I } \\
1^{\mathrm{a}} \text { vez } \\
\text { Grupo II } \\
1^{\mathrm{a}} \text { vez } \\
2^{\mathrm{a}} \text { vez }\end{array}$ & $\begin{array}{l}160 * / 6 \mathrm{~min} \\
160 * / 20 \mathrm{~min} \\
400 * / 15 \mathrm{~min}\end{array}$ & $\begin{array}{c}781,87 \times 10^{6} \mu \mathrm{L} \\
1.986,87 \times 10^{6} \mu \mathrm{L}\end{array}$ & Corazón & Nagata et al 2010 \\
\hline Sin reporte & Cit $\mathrm{Na} 3,8 \%$ & $320.133 \mu \mathrm{L}$ & $\begin{array}{l}1^{\text {a }} \text { vez } \\
2^{\text {a }} \text { vez }\end{array}$ & $\begin{array}{l}2.400 / 8 \mathrm{~min} \\
2.400 / 5 \mathrm{~min}\end{array}$ & $807,56 \times 10^{3} \mu \mathrm{L}$ & Vena auricular & Ohba et al 2012 \\
\hline
\end{tabular}

*Gravedades $(g)$ o fuerzas relativas de centrifugación (RCF). 
clínica no tiene correlación con la concentración plaquetas (Carmona y col 2011).

La presencia, tipo y cantidad de leucocitos concentrados en los PRP ha sido controvertida en medicina humana y equina (Carmona y col 2011, Giovanini y col 2012). Algunos autores consideran que la presencia de un número mayor de 5 veces la cantidad basal de WBC $\left(10^{3} / \mu \mathrm{L}\right)($ L-PRP) puede beneficiar los procesos de regeneración de tejidos, gracias al incremento de la liberación de factores de crecimiento (Ehrenfest y col 2009). Sin embargo, otros investigadores implican a los leucocitos en la formación de fibrosis y aumento de la inflamación (Chizzolini y col 2011). Hasta ahora no se ha dilucidado cuál de estas dos clase de PRP puede potencialmente presentar mejores efectos en la reparación de tejidos con los menores efectos secundarios, tales como inflamación y lisis celular.

En nuestro estudio obtuvimos una concentración de plaquetas para P-PRP-A de 186,9\% y para P-PRP-B de $164 \%$ mayor que el obtenido en sangre entera. Esta concentración fue mayor a la obtenida por simple centrifugación en caninos (Silva y col 2011ª), la cual fue de 149,1\% y menor que la de felinos (Silva y col 2011 $1^{\mathrm{b}}$ ) que fue del $247,7 \%$. Respecto a lo obtenido por los métodos de doble centrifugación en equinos de 172\% (Arguelles y col 2006) fue similar, pero la concentración fue menor que la obtenida en bovinos de $229 \%$. El número de células blancas concentradas en PRP de conejo fue de 0,98 veces menos para P-PRP-A y de 0,53 veces menor para la fracción P-PRP$\mathrm{B}$, mucho más bajo que lo encontrado en bovinos (López y col 2012) y equinos (Arguelles y col 2006), en los cuales se incrementó 1,57 y 1,18 veces respectivamente sobre valor basal. La concentración de células blancas obtenidas también por simple centrifugación en caninos (Silva y col $2011^{\mathrm{a}}$ ) fue de 0,57 y en felinos (Silva y col 2011 ${ }^{\mathrm{b}}$ ) de 0,36 menos que el valor basal, similar a lo obtenido para la fracción P-PRP-B de los conejos de este estudio.

La eficiencia de colección de plaquetas fue para la fracción P-PRP-A de $20,76 \%$ y para la P-PRP-B de $18,27 \%$, similar a la de $19,1 \%$ conseguida por doble centrifugación en el bovino (López y col 2012) y más alta que la obtenida en equino de 7,5\% (Arguelles y col 2006). Frente a la eficiencia de concentración obtenida en los felinos y caninos (Silva y col 2011ª) por centrifugación simple, fue mucho más baja, ya que en estas especie se obtuvieron eficiencias del $50 \%$ y $29,9 \%$ respectivamente.

Respecto a la integridad de las plaquetas, no se evidenció alteración morfológica según los parámetros MPV(fL) y PDW(\%) en ambas fracciones. Esto puede indicar que no se presentó activación temprana de las mismas con baja probabilidad de liberación de sus factores de crecimiento. Aunque sin diferencia estadísticamente significativa, el MPV(fL) se incrementó levemente en la fracción P-PRP-B de las hembras de este estudio. El aumento del valor de este parámetro está asociado con activación temprana de las plaquetas con la consecuente liberación de factores de crecimiento, sin embargo, el
MPV(fL) también puede estar elevado por la presencia de un mayor número de plaquetas nuevas circulantes y por el género, ya que en seres humanos se ha visto que las mujeres presentan un MPV(fL) aumentado en comparación con el valor encontrado en los hombres (Bain 1996). Es posible que este mismo fenómeno se haya podido presentar en las hembras leporinas de este estudio.

El PDW(\%) (volumen de distribución plaquetario) fue mayor en el hemograma de machos. Este parámetro plaquetario muestra las diferencias de tamaño entre plaquetas y la formación de seudópodos, por ello se usa como indicador de la activación de las mismas. A pesar de los incrementos por género, los parámetros no tuvieron diferencia estadística entre el hemograma y las fracciones P-PRP-A y P-PRP-B. La correlación negativa moderada $\left(\mathrm{R}_{\mathrm{S}}=-0,731\right) \mathrm{PLT}\left(10^{3} / \mu \mathrm{L}\right)$ y PDW $(\%)$, permite suponer que si las plaquetas de conejos se activan de forma temprana, la cantidad total de plaquetas en ambas fracciones será menor. Podemos concluir que con el método manual de centrifugación simple empleado en este estudio, se pueden producir P-PRP-B y P-PRP-A a partir de sangre de conejo citratada para su evaluación y potencial uso como biofármacos en distintas afecciones del aparato locomotor en humanos y otras especies. El tiempo de centrifugación corto y baja revolución, permiten obtener un producto de calidad por conservar la integridad de las plaquetas.

\section{AGRADECIMIENTOS}

Los autores agradecen a la Vicerrectoría de Investigaciones y Postgrados, Universidad de Caldas, Manizales, Colombia.

\section{REFERENCIAS}

Andres BM, GA Murrell. 2008. Treatment of tendinopathy: what works, what does not, and what is on the horizon. Clin Orthop Relat Res 466, 1539-1554.

Anitua E, I Andia, B Ardanza, P Nurden, AT Nurden. 2004. Autologous platelets as a source of proteins for healing and tissue regeneration. Thromb Haemost 91, 4-15.

Arguelles D, JU Carmona, J Pastor, A Iborra, L Vinals, P Martinez, E Bach, M Prades. 2006. Evaluation of single and double centrifugation tube methods for concentrating equine platelets. Res Vet Sci 81, 237-245.

Bain BJ. 1996. Ethnic and sex differences in the total and differential white cell count and platelet count. J Clin Pathol 49, 664-666.

Carmona JU, M Prades, D Argüelles. 2009. Concentrados autólogos de plaquetas como tratamiento de lesiones de tejidos blandos del aparato locomotor en caballos. Arch Med Vet 41, 77-82.

Carmona JU, C López, C Giraldo. 2011. Uso de concentrados autólogos de plaquetas como terapia regenerativa de enfermedades crónicas del aparato musculoesquelético equino. Arch Med Vet 43, 1-10.

Carpenter JE, KD Hankenson. 2004. Animal models of tendon and ligament injuries for tissue engineering applications. Biomaterials 25, 1715-1722. 
Chizzolini C, NC Brembilla, E Montanari, ME Truchetet. 2011. Fibrosis and immune dysregulation in systemic sclerosis. Autoimmun Rev 10, 276-281.

Dong Z, B Li, B Liu, S Bai, G Li, A Ding, J Zhao, Y Liu. 2012. Platelet-rich plasma promotes angiogenesis of prefabricated vascularized bone graft. J Oral Maxillofac Surg 70, 2191-2197.

Ehrenfest DMD, L Rasmusson, T Albrektsson. 2009. Classification of platelet concentrates: from pure platelet-rich plasma (P-PRP) to leucocyte- and platelet-rich fibrin (LPRF). Trends Biotechnol 27, 158-167.

Everts PAM, J Hoffmann, G Weibrich, CB Mahoney, JPAM Schönberger, A Van Zundert, JTA Knape. 2006. Differences in platelet growth factor release and leucocyte kinetics during autologous platelet gel formation. Transfus Med 16, 363-368.

Findikcioglu K, F Findikcioglu, R Yavuzer, C Elmas, K Atabay. 2009. Effect of platelet-rich plasma and fibrin glue on healing of critical-size calvarial bone defects. J Craniofac Surg 20, 34-40.

Giovanini AF, JR Grossi, CC Gonzaga, JC Zielak, I Gohringer, JD Vieira, J Kuczera, MA de Oliveira Filho, TM Deliberador. 2012. Leukocyte-Platelet-Rich Plasma (L-PRP) Induces an Abnormal Histophenotype in Craniofacial Bone Repair Associated with Changes in the Immunopositivity of the Hematopoietic Clusters of Differentiation, Osteoproteins, and TGF-beta1. Clin Implant Dent Relat Res, doi: 10.1111/j.1708-8208.2012.00478.x

Ishida K, R Kuroda, M Miwa, Y Tabata, A Hokugo, T Kawamoto, K Sasaki, M Doita, M Kurosaka. 2007. The regenerative effects of platelet-rich plasma on meniscal cells in vitro and its in vivo application with biodegradable gelatin hydrogel. Tissue Eng 13, 1103-1112.

Lee AJ, WH Chung, DH Kim, KP Lee, DJ Chung, SH Do, HY Kim. 2012. Anterior cruciate ligament reconstruction in a rabbit model using canine small intestinal submucosa and autologous platelet-rich plasma. J Surg Res 178, 206-215.

Lei H, L Gui, R Xiao. 2009. The effect of anticoagulants on the quality and biological efficacy of platelet-rich plasma. Clin Biochem 42, 1452-1460.

López C, C Giraldo, J Carmona. 2012. Evaluación de un método de doble centrifugación en tubo para concentrar plaquetas bovinas: estudio celular. Arch Med Vet 44, 109-115.

Lyras DN, K Kazakos, D Verettas, A Polychronidis, M Tryfonidis, S Botaitis, G Agrogiannis, C Simopoulos, A Kokka, E Patsouris. 2009. The influence of platelet-rich plasma on angiogenesis during the early phase of tendon healing. Foot Ankle Int 30, 1101-1106.

Lyras D, K Kazakos, D Verettas, A Polychronidis, C Simopoulos, S Botaitis, G Agrogiannis, A Kokka, E Patsouris. 2010. Immunohistochemical study of angiogenesis after local administration of platelet-rich plasma in a patellar tendon defect. Int Orthop 34, 143-148.

Masago H, Y Shibuya, S Munemoto, J Takeuchi, M Umeda, T Komori, Y Kuboki. 2007. Alveolar ridge augmentation using various bone substitutes - a web form of titanium fibers promotes rapid bone development. Kobe J Med Sci 53, 257-263.

Molloy T, Y Wang, G Murrell. 2003. The roles of growth factors in tendon and ligament healing. Sports Med 33, 381-394.

Nagata MJ, MR Messora, FA Furlaneto, SE Fucini, AF Bosco, VG Garcia, TM Deliberador, LG de Melo. 2010. Effectiveness of two methods for preparation of autologous platelet-rich plasma: an experimental study in rabbits. Eur $J$ Dent 4, 395-402.

Ohba S, W Wang, S Itoh, Y Takagi, A Nagai, K Yamashita. 2012. Acceleration of new bone formation by an electrically polarized hydroxyapatite microgranule/platelet-rich plasma composite. Acta Biomater 8, 2778-2787.

Rees JD, AM Wilson, RL Wolman. 2006. Current concepts in the management of tendon disorders. Rheumatology (Oxford) 45, 508-521.

Schnabel LV, HO Mohammed, BJ Miller, WG McDermott, MS Jacobson, KS Santangelo, LA Fortier. 2007. Platelet rich plasma (PRP) enhances anabolic gene expression patterns in flexor digitorum superficialis tendons. J Orthop Res 25, 230-240.

Silva R, C Rezende, F Paes-Leme, J Carmona. 2011ª Evaluación del método del tubo para concentrar plaquetas caninas: estudio celular. Arch Med Vet 43, 95-98.

Silva R, C Rezende, F Paes-Leme, J Carmona. 2011 $1^{\text {b }}$ Evaluación del método del tubo para concentrar plaquetas felinas: estudio celular. Arch Med Vet 43, 187-190.

Soomekh DJ. 2011. Current concepts for the use of platelet-rich plasma in the foot and ankle. Clin Podiatr Med Surg 28, 155-170.

Weibrich G, WK Kleis, R Buch, WE Hitzler, G Hafner. 2003. The Harvest Smart PRePTM system versus the FriadentSchutze platelet-rich plasma kit. Clin Oral Implants Res 14, 233-239.

White JG, G Escolar. 2000. EDTA-induced changes in platelet structure and function: adhesion and spreading. Platelets $11,56-61$. 